\title{
The fission yeast Rpb4 subunit of RNA polymerase II plays a specialized role in cell separation
}

\author{
Nimisha Sharma $\cdot$ Samuel Marguerat $\cdot$ Surbhi Mehta $\cdot$ \\ Stephen Watt · Jürg Bähler
}

Received: 4 May 2006 / Accepted: 21 August 2006 / Published online: 14 September 2006

(C) Springer-Verlag 2006

\begin{abstract}
RNA polymerase II is a complex of 12 subunits, Rpb1 to Rpb12, whose specific roles are only partly understood. Rpb4 is essential in mammals and fission yeast, but not in budding yeast. To learn more about the roles of Rpb4, we expressed the rpb4 gene under the control of regulatable promoters of different strength in fission yeast. We demonstrate that below a critical level of transcription, Rpb4 affects cellular growth proportional to its expression levels: cells expressing lower levels of $r p b 4$ grew slower compared to cells expressing higher levels. Lowered $r p b 4$ expression did not affect cell survival under several stress conditions, but it caused specific defects in cell separation similar to sep mutants. Microarray analysis revealed that lowered $r p b 4$ expression causes a global reduction in gene expression, but the transcript levels of a distinct subset of genes were particularly responsive to changes in rpb4 expression. These genes show some overlap with those regulated by the Sep1-Ace2 transcriptional cascade required for cell separation. Most notably, the
\end{abstract}

Communicated by H. Ronne.

Electronic supplementary material Supplementary material is available in the online version of this article at http://dx.doi.org/ 10.1007/s00438-006-0161-5 and is accessible for authorized users.

N. Sharma $\cdot$ S. Mehta

University School of Biotechnology,

G.G.S. Indraprastha University, Kashmere Gate,

Delhi 110006, India

N. Sharma $\cdot$ S. Marguerat $\cdot$ S. Watt $\cdot$ J. Bähler $(\varangle)$

Cancer Research UK Fission Yeast Functional Genomics

Group, Wellcome Trust Sanger Institute, Hinxton,

Cambridge CB10 1HH, UK

e-mail: jurg@sanger.ac.uk gene expression signature of cells with lowered $r p b 4$ expression was highly similar to those of $m c s 6, p m h 1$, sep10 and sep15 mutants. Mcs6 and Pmh1 encode orthologs of metazoan TFIIH-associated cyclin-dependent kinase (CDK)-activating kinase (Cdk7-cyclin H-Mat1), while Sep10 and Sep15 encode mediator components. Our results suggest that Rpb4, along with some other general transcription factors, plays a specialized role in a transcriptional pathway that controls the cell cycle-regulated transcription of a specific subset of genes involved in cell division.

Keywords Transcription $\cdot$ S. pombe $\cdot$ Cell division . Growth

\section{Introduction}

RNA polymerase II (pol II) transcribes the coding genes, and its activity is the main target for transcriptional control in all eukaryotes. The pol II enzyme consists of 12 subunits, which are highly conserved during evolution. The specific functions of the smaller subunits such as Rpb4 are relatively poorly understood, and they can show differences between organisms. For example, Rpb4 is essential in mammals and fission yeast (Schizosaccharomyces pombe), but not in budding yeast (Saccharomyces cerevisiae). Here we use fission yeast as a model to learn more about particular roles of Rpb4 in genome-wide transcription.

Much of our current understanding of the mechanism of transcription and the transcription machinery comes from studies using budding yeast. The distantly related fission yeast provides a valuable complementary model system to dissect the functions of different 
components of the transcriptional apparatus, because its transcription mechanism is more similar to that of mammals in some respects. For example, the initiation of transcription in both mammalian cells and $S$. pombe occur $\sim 30$ bp downstream of the TATA box, whereas in $S$. cerevisiae this distance can vary between 40 and $120 \mathrm{bp}$ (Li et al. 1994). Moreover, transcriptional initiation from mammalian promoters introduced into $S$. pombe occurs at the same site as in mammalian cells (Toyama and Okayama 1990).

As in other eukaryotes, $S$. pombe pol II is a multisubunit enzyme containing the 12 subunits Rpb1 to Rpb12. The four core subunits, Rpb1, Rpb2, Rpb3 and Rpb11, are homologous to the $\beta^{\prime}, \beta, \alpha^{\prime}$ and $\alpha$ subunits, respectively, of the eubacterial RNA polymerase. Five subunits, $\mathrm{Rpb} 5,6,8,10$ and 12 , are shared by the three eukaryotic RNA polymerases I, II and III, whereas $\mathrm{Rpb} 4, \mathrm{Rpb} 7$ and $\mathrm{Rpb} 9$ are subunits specific to pol II (Mitsuzawa and Ishihama 2004). The functions of most of these smaller subunits are not well characterized.

Rpb4 and Rpb7 form a conserved heterodimer complex in archaebacteria, yeast, plants and humans (Choder 2004). Crystallographic studies of pol II and biochemical data provide evidence for a role of the $\mathrm{Rpb} 4 / 7$ heterodimer in transcription initiation and RNA binding in S. cerevisiae (Armache et al. 2005; Bushnell and Kornberg 2003; Edwards et al. 1991; Orlicky et al. 2001). S. pombe Rpb7 interacts with an RNA-binding protein, implying a role in the coupling of RNA processing to transcription, and it associates with glyceraldehyde-3-phosphate dehydrogenase and actin (Mitsuzawa et al. 2003, 2005).

The in vivo functions of Rpb4 have been characterized in S. cerevisiae, and several lines of evidence suggest that it plays an important role in the response and survival to stress (reviewed by Choder 2004). S. cerevisiae Rpb4 is also important for activated transcription from a subset of promoters and in carbon and energy metabolism at moderate temperatures (Pillai et al. 2001, 2003). Recent data show that it is required for the decay of a class of mRNAs whose products are involved in protein synthesis, and it interacts with subunits of the mRNA decay complex Pat1/Lsm1-7 that enhances decapping (Lotan et al. 2005).

We are only beginning to understand the role of the Rpb4 subunit in S. pombe: it binds to the TFIIF-interacting carboxyl-terminal domain (CTD) phosphatase Fcp1, and it has been proposed to play a role in the assembly of the Fcp1-pol II complex, thereby promoting CTD phosphorylation for the reutilization of pol II in a new cycle of transcription (Kimura et al. 2002). $S$. pombe Rpb4 exhibits features that resemble its budding yeast counterpart, while others place it closer to its orthologs in multicellular eukaryotes. It is essential for viability, whereas in $S$. cerevisiae it is not required for cell growth under optimal conditions (Sakurai et al. 1999; Choder 2004). S. pombe Rpb4 contains 135 amino acids, placing it closer in size to its counterparts in humans (142 amino acids) and plants (138 amino acids) than to $S$. cerevisiae (221 amino acids). Stoichiometric amounts of Rpb4 associate with pol II in $S$. pombe and higher eukaryotes, while the fraction of pol II molecules containing Rpb4 in optimally growing S. cerevisiae cells is only about $20 \%$. Finally, Rpb4 is more tightly associated with $S$. pombe pol II than with S. cerevisiae pol II (Sakurai et al. 1999).

Here, we provide evidence that Rpb4 influences the growth and gene expression of $S$. pombe cells in a dosedependent fashion under optimal conditions. We show that lower levels of $r p b 4$ expression do not affect the survival of cells under several stress conditions. Our results imply a particularly important role for Rpb4 in the expression and regulation of a subset of genes involved in cell separation. The defects in cell growth and separation as well as the gene expression signatures associated with low levels of $r p b 4$ expression are similar to the phenotypes and expression signatures of a range of transcriptional regulatory mutants with defects in cell separation.

\section{Materials and methods}

Strains and molecular genetic methods

The $S$. pombe strains used in this study are listed in Table 1. JB22 was the parental haploid strain used to generate the strains expressing rpb4 under the P3nmt1, P41nmt1 and P81nmt1 promoters (Basi et al. 1993). The strains were constructed by PCR using the pFA6akanMX6-P3/P41/P81nmt1 constructs as described (Bähler et al. 1998), and the kanamycin-resistant transformants were selected on KsNoT media (Linder et al. 2002). Recombinant DNA methods were performed as described (Sambrook et al. 1989) and standard methodology and media for the manipulation of $S$. pombe were used (Moreno et al. 1991).

Table 1 Strains used in this study

\begin{tabular}{lll}
\hline Strain & Relevant genotype & Source \\
\hline JB22 & $972 h^{-}$ & Leupold (1970) \\
JB394 & kanMX6-p3nmt1-rpb4 $h^{-}$ & This study \\
JB395 & KanMX6-p41nmt1-rpb4 $h^{-}$ & This study \\
JB396 & kanMX6-p81nmt1-rpb4 $h^{-}$ & This study \\
\hline
\end{tabular}


Growth experiments

Pre-cultures for the growth curves were made by inoculating the respective strains in Edinburgh Minimal Medium (EMM) without thiamine or with $15 \mu \mathrm{M}$ thiamine and growing the cultures for $\sim 17 \mathrm{~h}$ at $32^{\circ} \mathrm{C}$ with shaking. Subsequently, the pre-culture was diluted into $50 \mathrm{ml}$ of fresh EMM medium at an $\mathrm{OD}_{600}=0.05-0.1$ and allowed to grow at $32^{\circ} \mathrm{C}$ with shaking. Samples were removed at various timepoints thereafter for measuring ODs at $600 \mathrm{~nm}$, microscopic examination, and microarray hybridization experiments. The low OD measurements in cells expressing $r p b 4$ under the P41nmt1 and P81nmt1 promoters is not caused by the phenotype, because mutants with similar phenotypes such as sepl show strong increases in OD during growth (unpublished data).

\section{Quantitative RT-PCR experiments}

To measure rpb4 expression, the strains expressing $r p b 4$ under the three promoters were grown for $24 \mathrm{~h}$ in EMM medium at $32^{\circ} \mathrm{C}$ (promoter on) or cultivated for a further $21 \mathrm{~h}$ in the presence of $15 \mu \mathrm{M}$ thiamine (promoter off). RNA was isolated by phenol extraction, purified using the RNeasy kit (Qiagen), and treated with RNase-free DNase (Roche). The reverse transcription reactions were performed using Superscript III (Invitrogen). Real-time PCR was performed using the Brilliant ${ }^{\circledR}$ SYBR $^{\circledR}$ Green QPCR Core Reagent Kit (Stratagene) on an Mx3000P QPCR system (Stratagene) with primers specific for $r p b 4$ or $f b a l$ (control) (sequences available on request). Arbitrary expression units were calculated using a standard curve made from serial dilutions of fission yeast genomic DNA.

\section{Stress response assays}

Serial dilutions (1/10) of rpb4 strains generated in our study were spotted on EMM plates with or without $15 \mu \mathrm{M}$ thiamine. The plates were supplemented with $0.5 \mathrm{mM}$ hydrogen peroxide or different concentrations of sorbitol (1,2, 3 and $4 \mathrm{M})$ to test the response to oxidative and osmotic stress, respectively. These plates were incubated at $32^{\circ} \mathrm{C}$ for 2 days and photographed. The effect of temperature stress was tested by incubating the strains at $36^{\circ} \mathrm{C}$ for 2 days.

\section{Microscopy}

Unfixed cells were observed at 2-h intervals for $25 \mathrm{~h}$ by light microscopy using a Zeiss Axioscope fluorescence microscope set up for differential interference contrast (DIC) with a $40 \times$ objective and Axiovision digital imaging system. To stain the nuclei, cells were spread onto microscopic glass slides. Subsequently, the cells were fixed by heating at $70^{\circ} \mathrm{C}$ for $1 \mathrm{~min}$ and DAPI (4', $6^{\prime}$ diamidino-2-phenylindole) was added at a final concentration of $1 \mu \mathrm{g} / \mathrm{ml}$. To view the division septa, calcofluor was added to cultures at a final concentration of $5 \mathrm{mg} / \mathrm{ml}$ and the cultures were incubated in the dark at room temperature for $5 \mathrm{~min}$. The cells were washed twice with phosphate buffer saline, and $5 \mu \mathrm{l}$ of cell suspension was spotted onto microscopic glass slides. DAPI- and calcofluor-treated cells were visualized under a Zeiss Axioscope microscope as above.

\section{Microarray experiments}

To compare the gene expression profiles as a function of $r p b 4$ expression, cells were grown in the presence of thiamine to $\mathrm{OD}_{600}$ of 0.2 , and $50 \mathrm{ml}$ of culture was centrifuged. The remaining culture was washed four to five times with EMM and subsequently grown in EMM medium without thiamine for $17 \mathrm{~h}$ and centrifuged. To identify transcripts affected by lowered $r p b 4$ expression, the desired strains were grown in the presence of thiamine for 21 and $23 \mathrm{~h}$ and harvested. A wild-type parental strain grown in the presence of thiamine served as a reference. Although thiamine leads only to minimal expression changes (Jenkins et al. 2005), this experimental design corrects for effects caused by the presence of thiamine. Pellets were frozen in liquid nitrogen and kept at $-70^{\circ} \mathrm{C}$ until further processing. RNA was isolated by phenol extraction and purified by the RNeasy kit (Qiagen). cDNA probes were prepared with Superscript (Invitrogen) and labeled with Cy3 or Cy5. Detailed methods were as described (Lyne et al. 2003; http://www.sanger.ac.uk/PostGenomics/ $S_{-}$pombe). Microarrays were scanned with a Genepix 4000B scanner and analyzed with Genepix software (Axon Instruments). Data filtering, normalization and quality control were performed as described (Lyne et al. 2003). The data are based on two to three independent biological repeats with dye swaps. To quantify global effects on gene expression, we repeated microarray analysis spiked with external control RNAs. The expression data were normalized using Bacillus subtilis spikes added in equal quantities to the RNA samples before labeling (Lee et al. 2005). Each microarray contains 720 control elements for the spikes, which are spread across the complete grid of the array in a spatially even manner. Clustering and visualization were done with GeneSpring. Gene annotations were taken from the $S$. pombe GeneDB database (www.genedb.org/ 
genedb/pombe/index.jsp). Overlaps between different gene lists were calculated using the hypergeometric distribution. All normalized data sets are available from our website: http://www.sanger.ac.uk/PostGenomics/ S_pombe.

\section{Results}

Dosage-dependent effect of Rpb4 on cell growth

$\mathrm{Rpb} 4$ is an essential subunit of $S$. pombe pol II. To elucidate its in vivo function(s), we constructed haploid strains expressing $r p b 4$ from its normal chromosomal location using the thiamine-regulated $n m t 1$ promoter. Three different versions of this promoter were used: the wild-type P3nmt1 promoter has the highest level of promoter activity and maintains substantial basal levels of expression even under repressed conditions; the two derivatives, P41nmt1 (medium strength) and P81nmt1 (weakest strength) have reduced levels of activity due to mutations in the TATA box (Basi et al. 1993).

We performed quantitative PCR of cells expressing $r p b 4$ under the three promoters, both in the absence ( $n m t 1$ promoter 'on') or presence (nmt1 promoter 'off') of thiamine to verify changing levels of $r p b 4$ mRNA (Fig. 1). Expression levels of rpb4

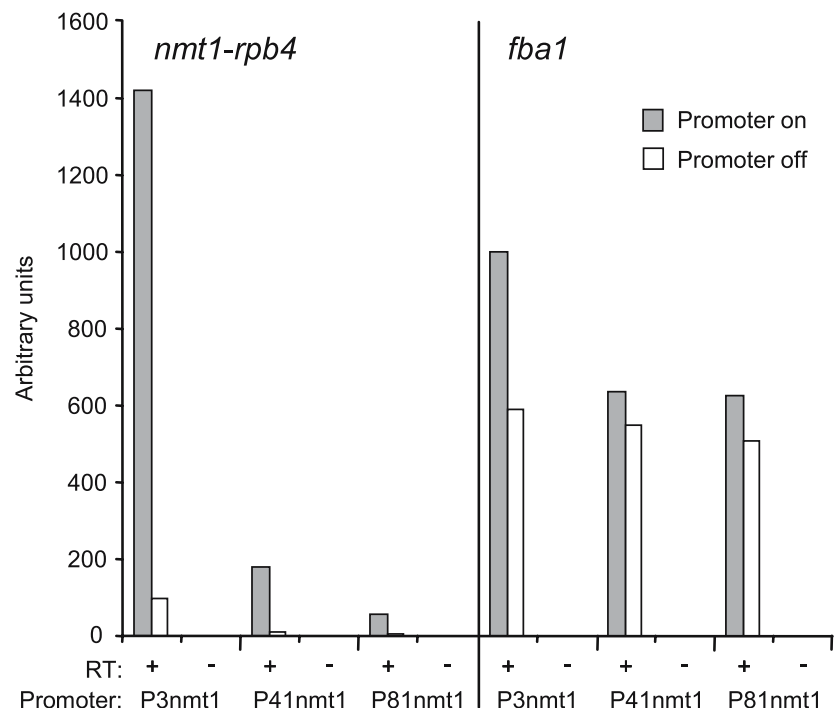

Fig. 1 Expression levels of $r p b 4$ mRNA when transcribed from different promoter constructs. Cells expressing $r p b 4$ from the $\mathrm{P} 3 n m t 1, \mathrm{P} 41 n m t 1$ or P81nmt1 promoter were grown for $24 \mathrm{~h}$ in EMM medium at $32^{\circ} \mathrm{C}$ (promoter on; gray bars) or cultivated for a further $21 \mathrm{~h}$ in the presence of thiamine (promoter off; white bars). rpb4 (left) and fbal (right; control) mRNA levels from the same cells were measured by quantitative RT-PCR. The PCR was performed on reverse transcription reactions, which were carried out in the presence $(\mathrm{RT}+)$ or absence $(\mathrm{RT}-$ ) of reverse transcriptase showed a range of more than 1,000-fold in the different conditions, and the relative expression levels were as expected for the three promoters of different strength. The rpb4 levels were especially low when expressed from the P41nmt1 and P81nmt1 promoters in the presence of thiamine. The fbal gene is highly expressed and was used as a control gene. Although it shows much less variation in expression levels than $r p b 4$, there are some changes in accordance with rpb4 levels (Fig. 1). This probably reflects effects on global transcription as a function of $r p b 4$ expression levels (see below).

To analyze effects of $r p b 4$ expression levels, the three strains were grown at $32^{\circ} \mathrm{C}$ either in the absence or presence of thiamine, and cell growth was monitored. In the absence of thiamine, all strains grew similar to each other and to the wild-type strain (Fig. 2a). This shows that rpb4 expressed from the weak P81nmt1 promoter under induced conditions was sufficient to sustain normal cell growth. When these strains were grown in the presence of thiamine, however, there was a direct correlation between residual promoter activity and growth rate: cells expressing rpb4 from the P $3 n m t 1$ promoter showed the same growth rate as wild-type cells, while cells expressing rpb4 from the P41nmt1 promoter and, even more so, from the P81nmt1 promoter showed reduced growth rates (Fig. $2 b$ ). Thus, in the presence of thiamine, the lowered Rpb4 levels become limiting for cell growth, and residual growth directly reflects basal expression levels from the different promoter constructs.

Effect of Rpb4 on cell growth under stress conditions

$\mathrm{Rpb} 4$ is required for stress response in $S$. cerevisiae (Choder 2004). We therefore, determined if Rpb4 played a similar role under stress conditions in S. pombe. Cells expressing varying levels of rpb4 were subjected to temperature stress $\left(36^{\circ} \mathrm{C}\right)$, osmotic stress ( $1 \mathrm{M}$ sorbitol) or oxidative stress $(0.5 \mathrm{mM}$ hydrogen peroxide). We found that neither in the presence nor absence of thiamine, the cells exhibited any increased stress sensitivity compared to control cells when grown under the different stress conditions (data not shown). Similar results were obtained when cells were grown in up to $2 \mathrm{M}$ sorbitol. No significant growth of either the wild-type or Rpb4 expressing cells was observed in the presence or absence of thiamine at 3 and $4 \mathrm{M}$ concentrations of sorbitol. These observations indicate that low levels of Rpb4 do not affect the survival of cells under any of the tested stress conditions. 


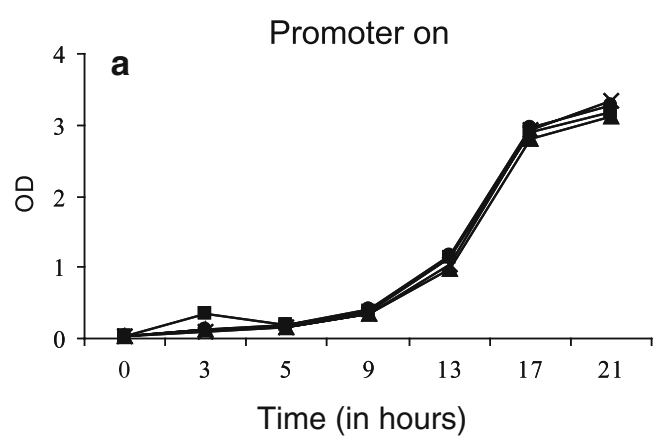

Fig. 2 Effect of Rpb4 on cell growth. Wild-type cells (filled circle) and cells expressing rpb4 from either the P3nmt1 (filled square), $\mathrm{P} 41$ nmt1 (filled triangle) or P81nmt1 (crosses) promoter were grown in EMM medium at $32^{\circ} \mathrm{C}$. $\mathrm{OD}_{600}$ was measured at the indi-

Repression of $r p b 4$ transcription causes defects in cell separation

We next examined whether different levels of $r p b 4$ expression would influence specific cellular processes. Cells expressing rpb4 from any of the three $n m t 1$ promoters in the absence of thiamine did not lead to any obvious phenotypes even after $25 \mathrm{~h}$ of growth in medium without thiamine (Fig. 3a).

In thiamine-containing medium, however, cells expressing $r p b 4$ from the weak P81nmt1 promoter became more elongated than the wild-type cells after $19 \mathrm{~h}$ of growth (Fig. 3b). Moreover, after continued incubation in the same medium for another $2 \mathrm{~h}(21 \mathrm{~h}$ with thiamine), cells also showed defects in cell separation, which became more evident after an additional $4 \mathrm{~h}$ of incubation ( $25 \mathrm{~h}$ with thiamine) (Fig. 3b). Most cells were elongated with either a single or multiple septa, and some cells also displayed bent morphologies and/or growth by branching. Cells expressing $r p b 4$ from the medium strength P41nmt1 promoter displayed no obvious cell separation defects until $23 \mathrm{~h}$ of growth in the presence of thiamine. Similar defects as described above, however, became apparent after $23 \mathrm{~h}$ of growth in thiamine (data not shown). In contrast, cells expressing rpb4 from the strong P3nmt1 promoter did not exhibit any aberrations in morphology or division after growth in thiamine-containing medium for $25 \mathrm{~h} \mathrm{(Fig.} \mathrm{3b)} \mathrm{and}$ continued to look like wild-type cells even at $40 \mathrm{~h}$ of growth in thiamine-containing medium (data not shown). Thus, the basal level of expression from the strongest promoter is sufficient to prevent the cell separation defects seen with the weaker promoters.

We investigated these phenotypes in more detail for cells expressing rpb4 under the P81nmt1 promoter by staining the division septa and cell nuclei (Fig. 3c).

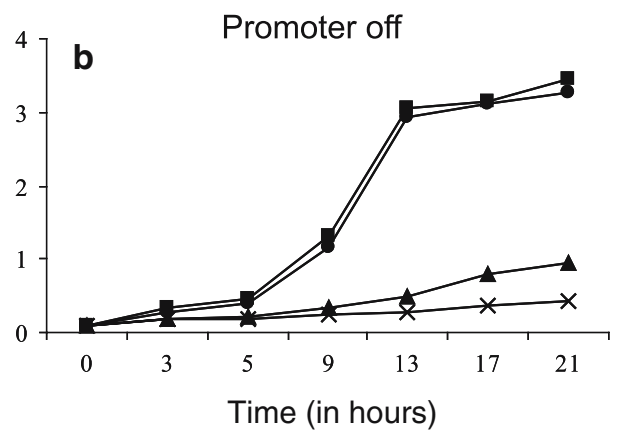

cated times. a In the absence of thiamine, all strains grew at similar rates. b In the presence of thiamine, the growth rates were dependent on the residual levels of $r p b 4$ being expressed from the different $n m t 1$ promoters

Several nuclei were present separated from each other by either a single septum or multiple, aberrant septa. Some cells also contained large deposits of septal material at random positions (Fig. 3c). We conclude that lowered rpb4 expression levels lead to filamentous growth with multiple cells remaining attached to each other. Similar phenotypes to those of cells expressing rpb4 under the P81nmt1 promoter have observed in several different mutants (see Discussion); for example, deletions in two transcription factor genes, sep1 and ace2, also lead to defects in cell separation (Fig. 3d; Bähler 2005). These findings raise the possibility that $\mathrm{Rpb} 4$ plays a specific role in cell separation after cytokinesis.

Low levels of $r p b 4$ transcripts most strongly affect expression of genes involved in septation

We used S. pombe whole-genome microarrays to investigate the effects on gene expression caused by low levels of rpb4. Wild-type cells and cells expressing rpb4 under the different regulatable promoters were grown in the presence and absence of thiamine followed by RNA extraction. Cells expressing rpb4 under the P3nmt1 promoter did not show any significant differences in gene expression profiles when compared with wild-type cells, either in the presence or absence of thiamine (data not shown), consistent with our earlier observations that these cells show normal growth and phenotypes. If $\mathrm{rpb} 4$ was expressed under the weakest promoter in the presence of thiamine, however, gene expression changed substantially. As down-regulation of rpb4 is expected to have a global effect on transcription, we used external spikes for normalization (Materials and methods). This revealed that mean transcript levels were $26-36 \%$ lower compared to wild-type cells 

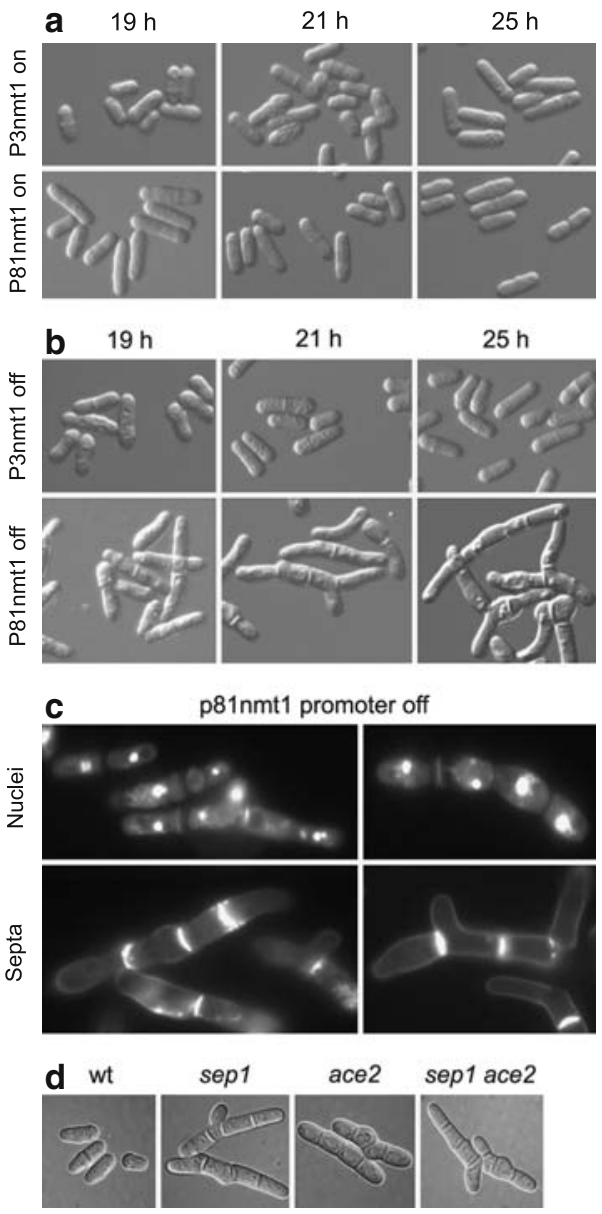

Fig. 3 Rpb4 affects cell separation and morphology. Cells expressing $r p b 4$ from either the $\mathrm{P} 3 n m t 1$ or P81nmt1 promoter were analyzed by microscopy. a Cells grown in EMM in the absence of thiamine (promoter 'on') at $32^{\circ} \mathrm{C}$ appear like normal, wild-type cells. b When grown in the presence of thiamine (promoter 'off'), cells expressing rpb4 from the P3nmt1 promoter appear normal, while those expressing rpb4 under the P81nmt1 promoter display defects in cell morphology and septation. Multiple phenotypes including elongated cells with single or multiple septa and growth by branching are evident. c Cells expressing $r p b 4$ under the P81nmt1 promoter were analyzed by fluorescence microscopy in the presence of thiamine. DAPI staining revealed single nuclei in each cell compartment, while calcofluor staining showed septa (some of them aberrant) separating the cell compartments. d Control cells and mutants showing similar phenotypes as cells expressing rpb4 under the P81nmt1 promoter cells. From left to right: wild-type cells, sep1 deletion mutants, ace 2 deletion mutants and sep1 ace 2 double deletion mutants

grown under the same conditions (Fig. 4a). The expression levels of rpb4 were two- to threefold lower under these conditions compared to the levels under its own promoter. Thus, lowered rpb4 expression leads to reduced overall transcript levels.

On top of this genome-wide down-regulation of transcription, 96 transcripts were at least 1.5 -fold upregulated in three of the four samples tested, while 150 genes were at least threefold downregulated in three of the four samples tested upon lowering rpb4 expression (Supplementary Tables S1 and S2). These genes were categorized according to their functions (Supplementary Table S3). The majority of the genes whose expression levels were affected by rpb4, encoded proteins involved in metabolism and transport. Many of the upregulated transcripts in cells with lowered rpb4 expression levels overlapped with genes upregulated during different environmental stresses (Chen et al. 2003) and nitrogen starvation (Mata et al. 2002) $\left(P \sim 1 \times 10^{-13}\right.$ to $\left.1 \times 10^{-36}\right)$. This probably reflects an indirect effect caused by the defects in these cells. A substantial number of the downregulated genes in the category of cell cycle and cell division played specific roles in cytokinesis and cell separation. For example, the two genes eng1 and agn1 encode $\beta$-glucanase and $\alpha$-glucanase, respectively; Eng1 degrades the primary division septum between the new ends of daughter cells and Agn1 hydrolyzes the old cell wall surrounding the septum leading to full separation of daughter cells (MartínCuadrado et al. 2003; Dekker et al. 2004). The genes adg1 and adg3 have also been shown to be involved in cell separation (Alonso-Nunez et al. 2005), and rng3 encodes a protein involved in formation of contractile ring during cytokinesis (Wong et al. 2000).

Both the lists of genes being upregulated and downregulated after rpb4 down-regulation showed highly significant overlaps with lists of genes upregulated or downregulated, respectively, in sep10, sep15, mcs6 and pmh1 mutants $\left(P \sim 1 \times 10^{-16}\right.$ to $\left.1 \times 10^{-47}\right)$ (Lee et al. 2005), whose genes encode different proteins involved in transcriptional regulation (see Discussion). Among the downregulated genes, the most pronounced overlap was observed with sep10 and sep15 mutants (Fig. 4b). The downregulated genes also showed some overlap with genes downregulated in sep1 and ace 2 mutants $\left(P \sim 1 \times 10^{-14}\right)$ (Rustici et al. 2004), which encode cell-cycle transcription factors. Figure $4 \mathrm{c}$ shows a cluster analysis of the genes most strongly regulated by $\mathrm{rpb} 4$ repression and their expression levels in the various transcriptional mutant backgrounds. The sep10, sep15, mcs6 and pmh1 mutants show highly similar expression signatures to cells with compromised rpb4 expression, while the overlap with sep1 and ace 2 mutants is less evident. Intriguingly, if rpb4 was overexpressed using the medium strength promoter, the expression levels of these genes were reversed, with downregulated genes becoming upregulated and upregulated genes becoming downregulated (Fig. 4c). Thus, transcript levels of this gene set are particularly responsive to the transcript levels of rpb4. 


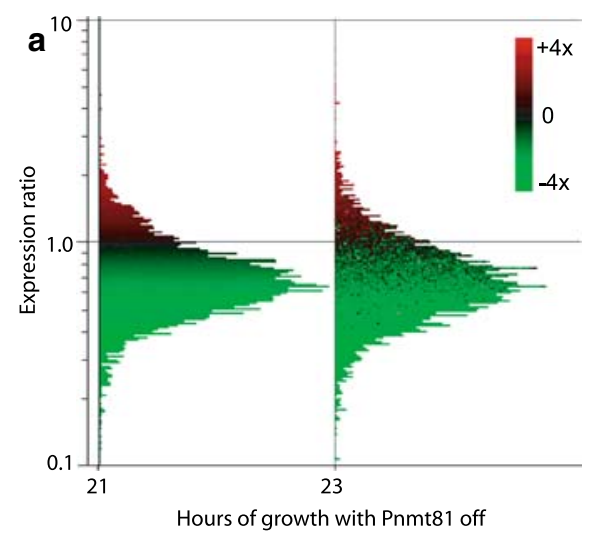

b
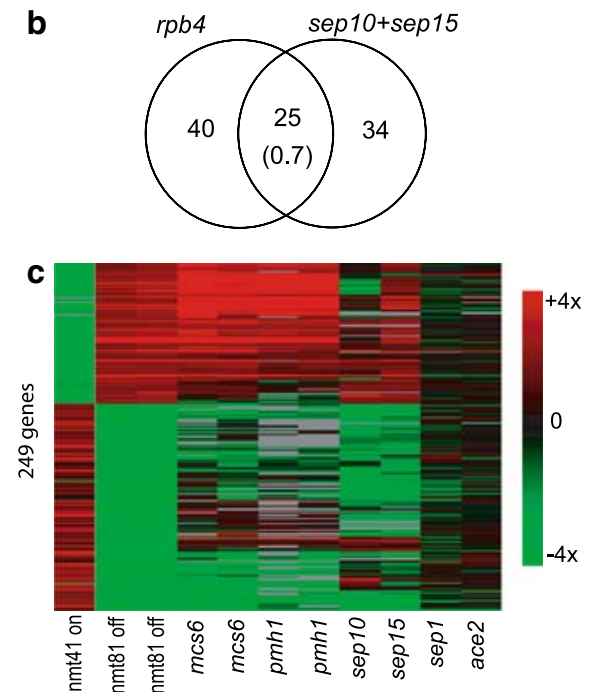

Fig. 4 Effects on global and specific gene expression as a function of $r p b 4$ levels. a Microarray experiments of cells expressing rpb4 under the weakest promoter. The histograms show the distribution of gene expression ratios of P81nmt1-rpb4 cells grown in the presence of thiamine for 21 and $23 \mathrm{~h}$ relative to wild-type cells grown in the presence of thiamine. Genes in both histograms are colore by their relative expression levels at $21 \mathrm{~h}$ as indicated at upper right. External spikes have been used for normalization, revealing a global down-regulation of transcript levels by $26 \%$ $(21 \mathrm{~h})$ to $36 \%(23 \mathrm{~h})$ on average when $r b p 4$ expression is lowered. The averages of two independent repeats for each timepoint are shown. b Overlap between the genes downregulated when rpb4 is expressed under the weakest promoter in the presence of thiamine and genes downregulated in both sep10 and sep15 mutants (Lee et al. 2005). The number in brackets represents the overlap expected by chance, given the sizes of the gene sets considered and the total number of 5,180 genes. The overlap is highly significant as calculated using the hypergeometric distribution $\left(P \sim 1 \times 10^{-30}\right)$. c Hierarchical cluster analysis of 249 genes that are either 1.5-fold upregulated or threefold downregulated when $r b p 4$ is expressed under the weakest promoter in presence of thiamine. Horizontal rows represent the profiles of hierarchically clustered genes. Columns represent experiments of different mutant strains and different time-points for the same strain. The transcript levels for each strain (indicated at bottom) relative to levels in wild-type cells are color-coded as indicated at right, and missing data are shown in gray. The microarray data from $m c s 6$, pmh1, sep10 and sep15 mutants are from Lee et al. (2005), and the data from sep1 and ace 2 mutants are from Rustici et al. (2004)

\section{Discussion}

We have analyzed functions of the Rpb4 subunit of pol II in $S$. pombe. Despite the overall conservation in structure and function of pol II across species, the $\mathrm{Rpb} 4$ subunit presents a special case. S. pombe Rpb4 is similar to its orthologs in higher eukaryotes with respect to its structure and essential nature (Sakurai et al. 1999), but in contrast to its human ortholog, it can form stable hybrid dimers with $S$. cerevisiae $\mathrm{Rpb} 7$ (Sakurai et al. 1999) and can rescue the temperaturesensitive phenotype associated with the lack of $S$. cerevisiae Rpb4 (Shpakovski et al. 2000). Thus, S. pombe Rpb4 may have intermediate characteristics useful to bridge the knowledge from $S$. cerevisiae and multicellular eukaryotes.

We used regulatable gene expression constructs to study the in vivo roles of Rpb4. Under optimal conditions, rpb4 influences the growth of cells in a dose-dependent manner when expression drops below a certain level using the regulatable promoters (Figs. 1, 2). In $S$. cerevisiae, deletion of the RPB4 gene leads to slow cell growth under optimal conditions (Woychik and Young 1989; Choder 2004), while in S. pombe rpb4 is essential (Sakurai et al. 1999). We found that low levels of $r p b 4$ expressed from the weakest promoter were sufficient for the cells to survive a range of different stress conditions including temperature stress. Accordingly, core stress genes were actually upregulated in $r p b 4$ mutants, and $S$. pombe Rpb4 is therefore unlikely to play any specialized role during environmental stress. S. cerevisiae Rpb4 is dispensable for oxidative and osmotic stress (Maillet et al. 1999), but unlike in $S$. pombe, it is required for survival of temperature stress (Woychik and Young 1989).

Fission yeast cells grown under low expression of $r p b 4$ were elongated and showed defects in cell separation as indicated by the accumulation of division septa, some of them highly aberrant (Fig. 3). Strains with cell separation defects similar to the ones described here include mutants in components of the exocyst complex (sec6, sec8, sec10 and exo70), an anillin homolog (mid2), septins (spn3, spn4), an endo- $\alpha$-1,3-glucanase (agn1), an endo- $\beta$-1,3-glucanase (eng1), calcineurin (ppb1), a MAP kinase and phosphatase (pmk1, pmp1), two transcription factors (sep1, ace2), and subunits of the mediator complex (sep10, sep11 and sep15) (Sipiczki et al.1993; Yoshida et al. 1994; Longtine et al. 1996; Toda et al.1996; Sugiura et al. 1998; Grallert et al. 1999; Wang et al. 2002; Martín-Cuadrado et al. 2003; Spåhr et al. 2003; Tasto et al. 2003; Dekker et al. 2004). In $S$. cerevisiae, haploid $r p b 4$ deletion mutants exhibit an axial budding pattern with normal yeast cell 
morphology, but diploid rpb4 mutants are more elongated than wild-type cells during nitrogen starvation and grow in a unipolar pattern, resembling pseudohyphae formation (Pillai et al. 2003).

Lowered expression of $r p b 4$ causes relatively modest reductions in global transcription, which probably leads to the reduced growth rates under these conditions (Fig. 4a). In addition, low rpb4 transcription results in substantially lowered expression of a distinct subset of genes. Among the strongly affected genes were eng1, agn1, adg1 and $a d g 3$ with known functions in cell separation, which are targets of a transcriptional cascade regulated by the Sep1 and Ace2 transcription factors (Rustici et al. 2004; Alonso-Nunez et al. 2005). Accordingly, the genes affected by lowered rpb4 expression significantly overlapped with genes affected in sep1 and ace 2 mutants. Much stronger similarities, however, were evident with the gene expression signatures of several strains mutated in the sep10, sep15, mcs6 and pmh1 genes, which encode various components of the transcriptional machinery. Sep10 and Sep15 are essential components of the mediator complex of pol II, while Mcs6 and Pmh1 are components of a complex homologous to metazoan Cdk7-cyclinHMat1, a cyclin-dependent kinase-activating kinase (CAK) and a pol II CTD kinase associated with transcription factor IIH (Spåhr et al. 2001, 2003; Lee et al. 2005). The overlap in expression signatures between these four mutants and cells with lowered rpb4 expression was evident for both upregulated and downregulated genes. These differentially regulated genes showed reverse regulation (upregulated genes became downregulated and vice versa) when $r p b 4$ was overexpressed from the medium strength promoter (Fig. 4c). Thus, the expression of this gene set is particularly responsive to $r p b 4$ expression levels and also depends on a range of other factors involved in general transcription. This could either reflect a specific transcriptional pathway involving Rpb4 and the other factors (either directly or indirectly via the control of a regulator), or the affected genes could be generally sensitive to transcriptional interference. In any case, these data show that the transcriptional pathway required for cell separation in fission yeast (Bähler 2005) is strongly affected when interfering with $r p b 4$ expression levels.

We speculate that the $\mathrm{Rpb} 4 / \mathrm{Rpb} 7$ complex is involved in linking the transcriptional output to the metabolic status of the cell. The rbp4 and $r p b 7$ transcripts are down-regulated when cells enter stationary phase (unpublished observations), Rpb7 interacts with the glycolytic enzyme glyceraldehyde-3-phosphate dehydrogenase (Mitsuzawa et al. 2005), and global transcription is sensitive to $r p b 4$ expression levels. It is possible that the transcriptional pathway required for cell separation is particularly sensitive for compromised transcription to adjust the growth mode to availability of nutrients: in abundant nutrients, global transcription is efficient and growth is best as single cells, while in limiting nutrients, global transcription and cell separation are compromised, and cells grow as multicellular pseudohyphae, which may allow a more efficient grazing for new nutrients as growth is directed. In the first description of $S$. pombe, multi-cellular pseudohyphae similar to those seen in rpb4 mutants have been observed (Lindner 1893); the natural capacity to switch to multi-cellular growth might have been lost in the yeast strains cultured in the laboratory.

Taken together our data suggest that $S$. pombe Rpb4, besides its general role in transcription, performs a more specialized function in regulating a specific gene expression program required for cell separation at the end of the cell cycle. It is possible that this role is carried out by interacting with other general factors of the transcriptional machinery such as the mediator and CTD kinase as suggested by similarities in phenotypes and expression signatures in our study. Accordingly, a comparison of the X-ray crystallographic structure of the complete pol II, including the $\mathrm{Rpb} 4 / 7$ heterodimer, with an electron microscopic structure of the pol II-mediator complex, has indicated that Rpb4/7 may interact with the mediator (Bushnell and Kornberg 2003). Alternatively, the subset of genes affected by changes in rpb4 expression (and by manipulating other general transcription factors) could reflect genes that are especially sensitive to transcriptional stress. This interpretation does not imply any specific gene expression program, although it could be used by the cell to adjust transcription to available nutrients. Given the conservation of Rpb4, it will be interesting to see whether the human ortholog plays a similar specialized role to transcribe particular subsets of genes.

Acknowledgments N.S. thanks all members of the Bähler laboratory for their help and advice, Derek Stemple and Nigel Carter and their group members for help with the use of microscopes, and the Sanger array facility for printing microarrays. N.S. was supported by a BOYSCAST fellowship from the Department of Science and Technology, Government of India, and S.M. holds a Fellowship for Advanced Researchers from the Swiss National Science Foundation. Research in the Bähler laboratory is funded by Cancer Research UK [CUK], grant number C9546/A5262.

\section{References}

Alonso-Nunez ML, An H, Martin-Cuadrado AB, Mehta S, Petit C, Sipiczki M, del Rey F, Gould KL, de Aldana CR (2005) Ace $2 p$ controls the expression of genes required for cell 
separation in Schizosaccharomyces pombe. Mol Biol Cell 16:2003-2017

Armache K-J, Mitterweger S, Meinhart A, Cramer P (2005) Structures of complete RNA polymerase II and its subcomplex Rpb4/7. J Biol Chem 280:7131-7134

Bähler J (2005) A transcriptional pathway for cell separation in fission yeast. Cell Cycle 4:39-41

Bähler J, Wu J-Q, Longtine MS, Shah NG, McKenzie A III, Steever AB, Wach A, Philippsen P, Pringle JR (1998) Heterologous modules for efficient and versatile PCR-based gene targeting in Schizosaccharomyces pombe. Yeast 14:943-951

Basi G, Schmid E, Maundrell K (1993) TATA box mutations in the Schizosaccharomyces pombe nmt1 promoter affect transcription efficiency but not the transcription start point or thiamine repressibility. Gene 123:131-136

Bushnell DA, Kornberg RD (2003) Complete, 12-subunit RNA polymerase II at 4.1-A resolution: implications for the initiation of transcription. Proc Natl Acad Sci USA 100:6969-6973

Chen D, Toone WM, Mata J, Lyne R, Burns G, Kivinen K, Brazma A, Jones N, Bähler J (2003) Whole-genome microarrays of fission yeast: characteristics, accuracy, reproducibility, and processing of array data. Mol Biol Cell 14:214-229

Choder M (2004) Rpb4 and Rpb7: subunits of RNA polymerase II and beyond. Trends Biochem Sci 29:674-681

Dekker N, Speijer D, Grun CH, van den Berg M, de Haan A, Hochstenbach F (2004) Role of the alpha-glucanase Agn1p in fission-yeast cell separation. Mol Biol Cell 15:3903-3914

Edwards AM, Kane CM, Young RA, Kornberg RD (1991) Two dissociable subunits of yeast RNA polymerase II stimulate the initiation of transcription at a promoter in vitro. J Biol Chem 266:71-75

Grallert A, Grallert B, Zilahi E, Szilagyi Z, Sipiczki M (1999) Eleven novel sep genes of Schizosaccharomyces pombe required for efficient cell separation and sexual differentiation. Yeast 15:669-686

Jenkins CC, Mata J, Crane RF, Thomas B, Akoulitchev A, Bähler J, Norbury CJ (2005) Activation of AP-1-dependent transcription by a truncated translation initiation factor. Eukaryot Cell 4:1840-1850

Kimura M, Suzuki H, Ishihama A (2002) Formation of a carboxyterminal domain phosphatase (Fcp1)/TFIIF/RNA polymerase II (pol II) complex in Schizosaccharomyces pombe involves direct interaction between Fcp1 and the Rpb4 subunit of pol II. Mol Cell Biol 22:1577-1588

Lee KM, Miklos I, Du H, Watt S, Szilagyi Z, Saiz JE, Madabhushi R, Penkett CJ, Sipiczki M, Bähler J, Fisher RP (2005) Impairment of the TFIIH-associated CDK-activating kinase selectively affects cell cycle-regulated gene expression in fission yeast. Mol Biol Cell 16:2734-2745

Leupold U (1970) Genetical methods for Schizosaccharomyces pombe. Methods Cell Physiol 4:169-177

Li Y, Flanagan PM, Tschochner H, Kornberg RD (1994) RNA polymerase II initiation factor interactions and transcription start site selection. Science 263:805-807

Linder K, Gregán J, Montgomery S, Kearsey SE (2002) Essential role of MCM proteins in premeiotic DNA replication. Mol Biol Cell 13:435-444

Lindner P (1893) Schizosaccharomyces pombe n. Sp., ein neuer Gährungserreger. Wochenschr Brauerei 10:1298-1300

Longtine MS, DeMarini DJ, Valencik ML, Al-Awar OS, Fares H, Virgilio CD, Pringle JR (1996) The septins: roles in cytokinesis and other processes. Curr Opin Cell Biol 8:106-119

Lotan R, Bar-On VG, Harel-Sharvit L, Duek L, Melamed D, Choder M (2005) The RNA polymerase II subunit Rpb4p mediates decay of a specific class of mRNAs. Genes Dev 19:3004-3016
Lyne R, Burns G, Mata J, Penkett CJ, Rustici G, Chen D, Langford C, Vetrie D, Bähler J (2003) Whole-genome microarrays of fission yeast: characteristics, accuracy, reproducibility, and processing of array data. BMC Genomics 4:27

Maillet I, Buhler JM, Sentenac A, Labarre J (1999) Rpb4p is necessary for RNA polymerase II activity at high temperature. J Biol Chem 274:22586-22590

Martín-Cuadrado AB, Dueñas E, Sipiczki M, Vázquez de Aldana CR, Del Rey F (2003) The endo-beta-1,3-glucanase eng1p is required for dissolution of the primary septum during cell separation in Schizosaccharomyces pombe. J Cell Sci 116:1689-1698

Mata J, Lyne R, Burns G, Bähler J (2002) The transcriptional program of meiosis and sporulation in fission yeast. Nat Genet 32:143-147

Mitsuzawa H, Ishihama A (2004) RNA polymerase II transcription apparatus in Schizosaccharomyces pombe. Curr Genet 44:287-294

Mitsuzawa H, Kanda E, Ishihama A (2003) Rpb7 subunit of RNA polymerase II interacts with an RNA-binding protein involved in processing of transcripts. Nucl Acids Res 31:46964701

Mitsuzawa H, Kimura M, Kanda E, Ishihama A (2005) Glyceraldehyde-3-phosphate dehydrogenase and actin associate with RNA polymerase II and interact with its Rpb7 subunit. FEBS Lett 579:48-52

Moreno S, Klar A, Nurse P (1991) Molecular genetic analysis of fission yeast Schizosaccharomyces pombe. Methods Enzymol 194:795-823

Orlicky SM, Tran PT, Sayre MH, Edwards AM (2001) Dissociable Rpb4-Rpb7 subassembly of RNA polymerase II binds to single-strand nucleic acid and mediates a post-recruitment step in transcription initiation. J Biol Chem 276:10097-10102

Pillai B, Sampath V, Sharma N, Sadhale P (2001) Rpb4, a nonessential subunit of core RNA polymerase II of Saccharomyces cerevisiae is important for activated transcription of a subset of genes. J Biol Chem 276:30641-30647

Pillai B, Verma J, Abraham A, Francis P, Kumar Y, Tatu U, Brahmachari SK, Sadhale PP (2003) Whole genome expression profiles of yeast RNA polymerase II core subunit, Rpb4, in stress and nonstress conditions. J Biol Chem 278:33393346

Rustici G, Mata J, Kivinen K, Lió P, Penkett CJ, Burns G, Hayles J, Brazma A, Nurse P, Bähler J (2004) Periodic gene expression program of the fission yeast cell cycle. Nat Genet 36:809-817

Sakurai H, Mitsuzawa H, Kimura M, Ishihama A (1999) The $\mathrm{Rpb} 4$ subunit of fission yeast Schizosaccharomyces pombe RNA polymerase II is essential for cell viability and similar in structure to the corresponding subunits of higher eukaryotes. Mol Cell Biol 19:7511-7518

Sambrook J, Fritsch EF, Maniatis T (1989) Molecular cloning: a laboratory manual, 2nd edn. Cold Spring Harbor Laboratory, Cold Spring Harbor

Shpakovski GV, Gadal O, Labarre-Mariotte S, Lebedenko EN, Miklos I, Sakurai H, Proshkin SA, Van Mullem V, Ishihama A, Thuriaux P (2000) Functional conservation of RNA polymerase II in fission and budding yeasts. J Mol Biol 295:11191127

Sipiczki M, Grallert B, Miklos I (1993) Mycelial and syncytial growth in Schizosaccharomyces pombe induced by novel septation mutations. J Cell Sci 104:485-493

Spåhr H, Samuelsen CO, Baraznenok V, Ernest I, Huylebroeck D, Remacle JE, Samuelsson T, Kieselbach T, Holmberg S, Gustafsson CM (2001) Analysis of Schizosaccharomyces 
pombe mediator reveals a set of essential subunits conserved between yeast and metazoan cells. Proc Natl Acad Sci USA 98:11985-11990

Spåhr H, Khorosjutina O, Baraznenok V, Linder T, Samuelsen CO, Hermand D, Makela TP, Holmberg S, Gustafsson CM (2003) Mediator influences Schizosaccharomyces pombe RNA polymerase II-dependent transcription in vitro. J Biol Chem 278:51301-51306

Sugiura R, Toda T, Shuntoh H, Yanagida M, Kuno T (1998) pmp1+, a suppressor of calcineurin deficiency, encodes a novel MAP kinase phosphatase in fission yeast. EMBO J 17:140-148

Tasto JJ, Morrell JL, Gould KL (2003) An anillin homologue, Mid2p, acts during fission yeast cytokinesis to organize the septin ring and promote cell separation. J Cell Biol 160:1093-1103

Toda T, Dhut S, Superti-Furga G, Gotoh Y, Nishida E, Sugiura $\mathrm{R}$, Kuno T (1996) The fission yeast pmk1+ gene encodes a novel mitogen-activated protein kinase homolog which regulates cell integrity and functions coordinately with the protein kinase C pathway. Mol Cell Biol 16:6752-6764
Toyama R, Okayama H (1990) Human chorionic gonadotropin alpha and human cytomegalovirus promoters are extremely active in the fission yeast Schizosaccharomyces pombe. FEBS Lett 268:217-221

Wang H, Tang X, Liu J, Trautmann S, Balasundaram D, McCollum D, Balasubramanian MK (2002) The multiprotein exocyst complex is essential for cell separation in Schizosaccharomyces pombe. Mol Biol Cell 13:515-529

Wong KC, Naqvi1 NI, Iino Y, Yamamoto M, Balasubramanian MK (2000) Fission yeast Rng3p: an UCS-domain protein that mediates myosin II assembly during cytokinesis. J Cell Sci 113:2421-2432

Woychik N, Young RA (1989) RNA polymerase II subunit RPB4 is essential for high- and low-temperature yeast cell growth. Mol Cell Biol 9:2854-2859

Yoshida T, Toda T, Yanagida M (1994) A calcineurin-like gene ppb1+ in fission yeast: mutant defects in cytokinesis, cell polarity, mating and spindle pole body positioning. J Cell Sci 107:1725-1735 